\title{
Spectrum and Antibiotic Resistance of Uropathogens Isolated from Hospital and Community Patients with Urinary Tract Infections in Two Large Hospitals in Kuwait
}

\author{
Noura Al Sweih ${ }^{a}$ Wafaa Jamal $^{\mathrm{b}}$ V.O. Rotimi ${ }^{\mathrm{a}}$ b

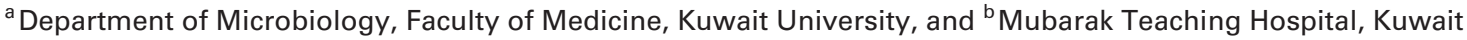

\section{Key Words}

Urinary tract infection - Uropathogens · Antibiotic resistance

\begin{abstract}
Objectives: To determine the spectrum of microbial etiology and antibiotic resistance pattern of the uropathogens that cause urinary tract infections in 2 large teaching hospitals in Kuwait over a period of 1 year. Materials and Methods: The Vitek identification card system was used to identify the uropathogens. Susceptibility of the isolates against 18 antibiotics was performed by the microbroth dilution method using the Vitek automated system. In addition, gram-positive bacteria were tested in parallel by the disk diffusion technique. Results: The six overall most common isolates were: Escherichia coli, accounting for $47 \%$ of isolates in both hospitals, followed by Candida spp. (10.8\%), Klebsiella pneumoniae (9.6\%), Streptococcus agalactiae (GBS; 9.5\%), Enterococcus faecalis (4.2\%) and Pseudomonas aeruginosa (4.1\%). Amikacin provided the widest coverage amongst all the antibiotics tested followed by ciprofloxacin, gentamicin and piperacillin-tazobactam. For the gram-negatives, high resistance $(26-63 \%)$ to the $\beta$-lactam antibiotics was noted, especially to ampicillin, amoxicillin-clavulanic
\end{abstract}

acid, cephalothin and cefuroxime. Resistance to trimethoprim-sulfamethoxazole was also high. None of the enterococci was resistant to the glycopeptides, but 38$60 \%$ of the Staphylococcus haemolyticus were resistant to vancomycin or teicoplanin. Conclusion: These data show the high level of antimicrobial resistance amongst the uropathogens causing urinary tract infection in the two hospitals studied.

Copyright $(2005$ S. Karger AG, Basel

\section{Introduction}

Urinary tract infection (UTI) is a very common infection both in the community and hospital patients and ranks high amongst the most common reasons that compel a patient to seek medical attention [1]. Acute UTIs are associated with substantial morbidity and problems of recurrent infections. Studies have shown that approximately $25 \%$ of women with first UTI will have another attack within 6 months [2]. A mounting body of evidence indicate that UTI is responsible for a large proportion of antibiotic consumption in and out of the hospital. No wonder that the infection has a large socio-economic impact and contributes to the emergence of antibiotic resistance in the hospital and the community [3,4].

\section{KARGER}

Fax +4161306 1234 E-Mail karger@karger.ch www.karger.com
(C) 2005 S. Karger AG, Basel

$1011-7571 / 05 / 0146-0401 \$ 22.00 / 0$

Accessible online at:

www.karger.com/mpp
Dr. Noura Al Sweih

Department of Microbiology

Faculty of Medicine, Kuwait University

PO Box 24923, Safat 13110 (Kuwait)

Tel. +965 5312300, ext. 6062, Fax +965 5332719, E-Mail nalsweih@hsc.edu.kw 
To optimize the use of empiric antibiotic therapy for UTI, it is important for clinicians to have a working knowledge of the etiological agents and the susceptibility patterns of UTI pathogens in their population. Since uropathogens and their susceptibility may vary from time to time and from one institution to another, each hospital or institution must have its own evaluation for such infection so that any change in the causative agents and susceptibility pattern can be detected and managed accordingly. Such evaluation is also useful to detect the spread of infection from one patient to another in the hospital as well as to assist in the setting up of an antibiotic policy.

Several studies in the past have shown that Escherichia coli is the most common etiological agent of UTI in hospital and community patients and that hospital-acquired UTI (HA-UTI), in particular, is characteristically associated with a higher prevalence of enterococci and coagulase-negative staphylococci (CNS) [5-9]. Of interest is the report of many recent studies from USA and Europe that showed increasing antibiotic resistance among uropathogenic $E$. coli to ampicillin, trimethoprim and sulfonamides $[6,7,9,10]$. Also a previous report from Kuwait reported high resistance rate to ampicillin by this organism [11]. Importantly, the majority of acute UTIs are caused by strains of these uropathogens [12]. The apparent shift in the etiological agents of different infections encountered in the last decade has not been assessed in UTI in this part of the world. It is noteworthy that no comparative information has emerged concerning this important infection and the problem of antibiotic resistance amongst its uropathogens encountered in different hospitals in the Gulf region, especially in Kuwait with its diverse population.

This study was designed to determine the microbial etiological agents of UTI, as well as their antimicrobial susceptibility, amongst the inpatients and outpatients of a maternity and a general hospital in Kuwait.

\section{Materials and Methods}

This study was conducted in the Departments of Microbiology of two large teaching hospitals (Mubarak Al-Kabeer Hospital, $\mathrm{MKH}$, and Maternity Hospital, $\mathrm{MH}$ ) attached to the Medical School of Kuwait University. MKH is a 500-bedded tertiary hospital situated adjacent to the Medical School with a general intensive care unit (ICU), a pediatric ICU, a busy urology unit, renal dialysis and kidney transplant units. MH, a specialized 400-bedded hospital, dealing with obstetric and gynecological patients as well as neonates, is $18 \mathrm{~km}$ away. It also has an adult ICU and a special care baby unit as well as neonatal ICU. A total of 37,203 and 14,359 records of urinary uropathogens and their antibiograms from $\mathrm{MKH}$ and $\mathrm{MH}$, respectively, for the study period of January to December 2002 , were analyzed. Urine specimens with bacterial growth of $\geq 10^{5}$ colony-forming unit $/ \mathrm{ml}$ were regarded as significant bacteriuria. The materials were divided into 2 groups. One group comprised urine samples from patients admitted to the hospitals (HAUTI) and the second group comprised samples from polyclinics and outpatient clinics attached to the hospitals (community-acquired UTI; CA-UTI).

Antibiotic susceptibility testing was performed for all significant gram-negative isolates by an automated Vitek machine (bioMérieux, Marcy-I'Etoile, France). All gram-positive bacteria were tested manually by Kirby-Bauer method. The results were interpreted according to the recommendation of the National Committee for Clinical Laboratory Standards [13]. Susceptibility testing for yeasts was not done. Identification and susceptibility testing were carried out by the same methods in both laboratories. In order to eliminate duplication, only one species per patient was included in the analysis. The materials were not stratified according to age or clinical diagnosis.

Reference strains of E. coli, ATCC 25922, and Pseudomonas aeruginosa, ATCC 27853, were used as controls for the gram-negative bacteria and were included in all daily runs. Staphylococcus aureus, ATCC 25923, and Enterococcus faecalis, ATCC 929212, were used as gram-positive control strains.

\section{Results}

Of the 37,203 urine samples from MKH, 3,594 (9.7\%) and of 14,359 from MH 1,102 (7.7\%) had significant bacteriuria. In order to eliminate duplicated results, only one species per patient was included in this analysis. The materials were not stratified according to age or clinical diagnosis.

The top six uropathogens causing UTI isolated from both hospitals were E. coli (47\%), Candida spp. (10.8\%), Klebsiella pneumoniae (9.6), Streptococcus agalactiae (GBS; 9.5\%), E. faecalis (4.2\%) and P. aeruginosa (4.1\%).

The distribution of gram-negative, gram-positive bacteria and yeast isolated from the clinical samples is shown in table 1. These isolates from both hospital laboratories represented clinically significant pathogens. As shown in this table, E. coli was the predominant pathogen isolated from urine samples from HA-UTI and CA-UTI patients in both hospitals. In $\mathrm{MKH}$, the top five most common organisms causing UTI in hospitalized patients were, in the descending order, the following: E. coli (35\%), Candida spp. (19.8\%), K. pneumoniae (9.9\%), P. aeruginosa $(9.8 \%)$ and E. faecalis (5.2\%). In the CA-UTI patients, they were: E. coli (56.7\%), S. agalactiae (group B streptococci; GBS) (10.4\%), K. pneumoniae (8.5\%), E. faecalis 
Table 1. Microbial uropathogens isolated from urine of patients with UTIs in MKH and MH

\begin{tabular}{|c|c|c|c|c|}
\hline \multirow[t]{3}{*}{ Microorganism } & \multicolumn{4}{|c|}{ Number (\%) of micro-organisms isolated from UTI } \\
\hline & \multicolumn{2}{|c|}{ MKH patients } & \multicolumn{2}{|l|}{ MH patients } \\
\hline & community & hospital & community & hospital \\
\hline \multicolumn{5}{|l|}{ Gram-negative bacteria } \\
\hline E. coli & $1,389(56.7)$ & $401(35)$ & $146(46.2)$ & $268(34.1)$ \\
\hline K. pneumoniae & $209(8.5)$ & $113(9.9)$ & $33(10.4)$ & $97(12.3)$ \\
\hline P. aeruginosa & $68(2.8)$ & $112(9.8)$ & $3(0.9)$ & $10(1.3)$ \\
\hline Acinetobacter calcoaceticus & $26(1.1)$ & $40(3.5)$ & $1(0.3)$ & $11(1.4)$ \\
\hline P. mirabilis & $49(2.0)$ & $14(1.2)$ & $4(1.3)$ & $2(0.3)$ \\
\hline Enterobacter spp. & $42(1.7)$ & $48(4.2)$ & $5(1.6)$ & $11(1.4)$ \\
\hline Citrobacter spp. & $10(0.4)$ & $14(1.2)$ & $4(1.3)$ & $2(0.3)$ \\
\hline Miscellaneous GNB & $56(2.3)$ & $25(2.2)$ & 0 & $32(4)$ \\
\hline \multicolumn{5}{|l|}{ Gram-positive bacteria } \\
\hline S. agalactiae (GBS) & $255(10.4)$ & $28(2.4)$ & $38(12)$ & $126(16.0)$ \\
\hline E. faecalis & $95(3.9)$ & $59(5.2)$ & $8(2.5)$ & $33(4.2)$ \\
\hline S. aureus & $32(1.3)$ & $11(1)$ & $2(0.6)$ & $4(0.5)$ \\
\hline S. epidermidis & $25(1)$ & $8(0.7)$ & 0 & 0 \\
\hline S. haemolyticus & $21(0.9)$ & $5(0.4)$ & 0 & 0 \\
\hline S. saprophyticus & $74(3)$ & $26(2.3)$ & $6(1.9)$ & 0 \\
\hline Miscellaneous GPB & $38(1.5)$ & $14(1.2)$ & $9(2.8)$ & $26(3.3)$ \\
\hline \multicolumn{5}{|l|}{ Yeasts } \\
\hline C. albicans & $22(0.9)$ & $63(5.5)$ & $45(14.2)$ & $135(17.2)$ \\
\hline Candida spp. & $38(1.6)$ & $164(14.3)$ & $12(3.8)$ & $29(3.7)$ \\
\hline Total & 2,449 & 1,145 & 316 & 786 \\
\hline
\end{tabular}

(3.9\%) and Staphylococcus saprophyticus (3\%). Among the HA-UTI patients at the MH, the commonest organisms were: E. coli (34.1\%), Candida spp. (20.9\%) of which, Candida albicans was $17.2 \%$, GBS (16\%), K. pneumoniae $(12.3 \%)$, E. faecalis (4.2\%) and S. saprophyticus (3.3\%). Those responsible for UTI in the community were: $E$. coli (46.2\%), Candida spp. (18\%), GBS (12\%) and K. pneumoniae (10.4\%).

\section{Susceptibility Data}

Of the antimicrobial agents tested at MKH (table 2), only amikacin, cefotaxime, ciprofloxacin and nitrofurantoin showed excellent activities against the $E$. coli isolates. Only 2 and $1 \%, 2$ and $5 \%, 5$ and $6 \%$ and 5 and $6 \%$ of the E. coli isolates from the CA-UTI and HA-UTI cases were resistant to amikacin, cefotaxime, ciprofloxacin and nitrofurantoin, respectively. Resistance rates by these isolates, from both CA-UTI and HA-UTI to ampicillin (63 and $76 \%$, respectively), amoxicillin-clavulanic acid (amoxiclav) (45 and 53\%), trimethoprim-sulfamethoxazole (SXT) (51 and 44\%), piperacillin (46 and 62\%) and nalidixic acid (16 and 25\%) were very high. With the ex- ception of Proteus mirabilis, the resistance rates of all the other gram-negative bacteria to the four first-line antibiotics (ampicillin, amoxiclav, SXT and nitrofurantoin) were also far above the acceptable 10\% level of resistance.

At $\mathrm{MH}$, the resistance rates of the gram-negative bacteria were relatively lower than the rates at MKH. Table 3 shows the antibiotic resistance rates of the gram-negative bacterial isolates. A large proportion of the E. coli isolates from the $\mathrm{MH}$ and $\mathrm{MKH}$ cases were also resistant to ampicillin (53 and 63\%, respectively), amoxiclav (26 and 26\%), SXT (42 and 37\%) and piperacillin (32 and $52 \%)$. However, only 4 and $3 \%$ of $E$. coli isolates from these two settings, respectively, were resistant to nitrofurantoin.

In general, $\beta$-lactam antibiotics, e.g. ampicillin and amoxicillin-clavulanic acid, were less active than the 2 nd and 3 rd generation cephalosporins against $E$. coli from both hospitals. In $\mathrm{MH}$ isolates of $E$. coli resistance rates to cefotaxime, cefuroxime and cephalothin were 3,20 and $9 \%$, respectively, whereas in the MKH isolates, 8,18 and $17 \%$, respectively, were resistant. 
Table 2. Percentage of resistant gram-negative uropathogens isolated from outpatients vs. inpatients with UTIs in MKH

\begin{tabular}{|c|c|c|c|c|c|c|c|c|c|c|c|c|c|c|c|}
\hline K. pneuтопiаe & 209/113 & $2 / 1$ & $25 / 35$ & $99 / 98$ & $26 / 26$ & $1 / 8$ & $1 / 17$ & $20 / 9$ & $22 / 33$ & $2 / 4$ & $10 / 14$ & $46 / 65$ & $3 / 9$ & $33 / 80$ & $0 / 41$ \\
\hline P. aeruginosa & $68 / 112$ & $10 / 12$ & - & - & - & - & - & $0 / 22$ & - & $14 / 24$ & $50 / 24$ & $-/-$ & $21 / 22$ & $3 / 17$ & $0 / 10$ \\
\hline A. calcoaceticus & $26 / 40$ & $31 / 54$ & $24 / 62$ & $88 / 92$ & 96/99 & $36 / 58$ & $80 / 72$ & $27 / 51$ & $19 / 34$ & $54 / 44$ & $25 / 44$ & $96 / 100$ & $33 / 58$ & $35 / 58$ & $19 / 34$ \\
\hline
\end{tabular}

$\mathrm{CA}=$ Community acquired $\mathrm{HA}=$ hospital acquired; $\mathrm{AN}=$ amikacin; $\mathrm{AUG}=$ amoxicillin/clavulanic acid $; \mathrm{AMP}=$ ampicillin $; \mathrm{CF}=$ cephalothin; $\mathrm{CTX}=$ cefotaxime; $\mathrm{CXM}$ = cefuroxime; $\mathrm{CIP}$ = ciprofloxacin; $\mathrm{SXT}$ = cotrimoxazole; $\mathrm{GN}$ = gentamicin; $\mathrm{NA}$ = nalidixic acid; $\mathrm{NIT}=$ nitrofurantoin; $\mathrm{NOR}=$ norfloxacin; $\mathrm{PIP}=$ piperacillin; $\mathrm{TZP}=$ piperacillin/tazobactam .

Table 3. Percentage of outpatients vs. inpatients with UTI gram-negative pathogens resistant to antibiotics in MH

\begin{tabular}{|c|c|c|c|c|c|c|c|c|c|c|c|c|c|c|}
\hline Bacteria & $\mathrm{CA} / / \mathrm{HA}$ & \multicolumn{13}{|c|}{ Percentage of CA vs. HA urinary bacterial isolates resistant to: } \\
\hline K. pneumoniae & 33/97 & $0 / 0$ & $3 / 20$ & $100 / 100$ & $0 / 7$ & $0 / 0$ & $3 / 4$ & $4 / 0$ & $16 / 12$ & $2 / 4$ & $0 / 0$ & $59 / 100$ & $32 / 30$ & $0 / 0$ \\
\hline$P$. aeruginosa & $3 / 10$ & $0 / 0$ & - & - & - & - & - & $0 / 0$ & - & $0 / 30$ & - & - & $0 / 0$ & $0 / 0$ \\
\hline P. mirabilis & $4 / 2$ & $0 / 0$ & $0 / 50$ & $0 / 33$ & $0 / 0$ & $0 / 0$ & $0 / 0$ & $0 / 0$ & $33 / 45$ & $0 / 0$ & $0 / 0$ & $100 / 100$ & $0 / 0$ & $0 / 0$ \\
\hline
\end{tabular}

$\mathrm{CA}=$ Community acquired; $\mathrm{HA}=$ hospital acquired; $\mathrm{AN}=$ amikacin; $\mathrm{AUG}=$ amoxicillin/clavulanic acid $; \mathrm{AMP}=$ ampicillin; $\mathrm{CF}=$ cephalothin; $\mathrm{CTX}=$ cefotaxime; $\mathrm{CXM}$ = cefuroxime; $\mathrm{CIP}$ = ciprofloxacin; $\mathrm{SXT}=$ cotrimoxazole; $\mathrm{GN}$ = gentamicin; NA = nalidixic acid; NIT = nitrofurantoin; PIP = piperacillin; $\mathrm{TZP}=$ piperacillin/tazobactam

Table 4. Percentage of resistant gram-positive uropathogens isolated from outpatients vs. inpatients with UTI in MKH and MH

\begin{tabular}{|c|c|c|c|c|c|c|c|c|c|c|}
\hline \multirow[t]{2}{*}{ Bacteria } & \multirow[t]{2}{*}{$\mathrm{CA} / \mathrm{HA}$} & \multicolumn{9}{|c|}{ Percentage of CA vs. HA uropathogens resistant to: } \\
\hline & & AMP & $\mathrm{CF}$ & $\mathrm{CC}$ & CLOX & SXT & $\mathrm{P}$ & VAN & TEIC & NIT \\
\hline \multicolumn{11}{|l|}{ MKH } \\
\hline S. agalactiae & $255 / 28$ & $0 / 0$ & - & - & - & - & $0 / 0$ & $0 / 0$ & $0 / 0$ & $0 / 0$ \\
\hline E. faecalis & $95 / 59$ & $2 / 26$ & - & - & - & - & $26 / 30$ & $0 / 0$ & $0 / 0$ & $1 / 0$ \\
\hline S. aureus & $32 / 11$ & - & $14 / 18$ & $12 / 33$ & $0 / 18$ & $7 / 36$ & $98 / 99$ & $0 / 0$ & $0 / 0$ & $0 / 0$ \\
\hline S. epidermidis & $25 / 8$ & - & $13 / 19$ & $20 / 25$ & $52 / 66$ & $37 / 41$ & $89 / 78$ & $0 / 0$ & $0 / 0$ & $25 / 16$ \\
\hline S. haemolyticus & $21 / 5$ & - & $33 / 80$ & $19 / 40$ & $14 / 20$ & $44 / 33$ & $76 / 80$ & $38 / 40$ & $47 / 60$ & $17 / 21$ \\
\hline S. saprophyticus & $74 / 26$ & - & $46 / 50$ & $0 / 0$ & $46 / 50$ & $8 / 50$ & $85 / 100$ & $0 / 0$ & $0 / 0$ & $15 / 0$ \\
\hline \multicolumn{11}{|l|}{$M H$} \\
\hline S. agalactiae & $38 / 126$ & $0 / 0$ & $0 / 0$ & $16 / 4$ & $-1-$ & $27 / 35$ & $0 / 0$ & $0 / 0$ & $0 / 0$ & $0 / 0$ \\
\hline E. faecalis & $8 / 33$ & $0 / 13$ & $-/-$ & $-/-$ & $-/-$ & $33 / 55$ & 13/91 & $0 / 0$ & $0 / 0$ & $38 / 49$ \\
\hline S. aureus & $2 / 4$ & $100 / 100$ & $0 / 0$ & $0 / 0$ & $0 / 0$ & $0 / 25$ & $100 / 100$ & $0 / 0$ & $0 / 0$ & $0 / 0$ \\
\hline S. saprophyticus & $6 / 0$ & $100 /-$ & $0 /-$ & $0 /-$ & $0 /-$ & $100 /-$ & $100 /-$ & $0 /-$ & $0 /-$ & $0 /-$ \\
\hline
\end{tabular}

$\mathrm{AMP}=$ Ampicillin CF = cephalothin $\mathrm{CC}=$ clindamycin CLOX = cloxacillin; $\mathrm{SXT}=$ cotrimoxazole $; \mathrm{P}=$ penicillin; $\mathrm{VAN}=$ vancomycin; TEIC = teicoplanin; NIT = nitrofurantoin . 
Ciprofloxacin activity against $E$. coli was fairly good; 8 and 7\% of the CA-UTI and HA-UTI isolates, respectively, were resistant. $P$. aeruginosa isolates were very sensitive; none was resistant to amikacin, ciprofloxacin and piperacillin.

The resistance rates of the gram-positive bacterial isolates to the commonly tested antibiotics in $\mathrm{MKH}$ are shown in table 4. E. faecalis had high resistance rates of 26 and 30\% in the CA-UTI and HA-UTI cases, respectively, to penicillin. The CNS exhibited high level of resistance to cephalothin, clindamycin, cloxacillin and penicillin.

As shown in table 4, none of the gram-positive bacteria isolated from UTI cases at MH were resistant to vancomycin or teicoplanin. All the GBS were sensitive to penicillin, and the entire $E$. faecalis isolates were sensitive to the glycopeptides. Cloxacillin resistance in $S$. aureus was observed more often in MKH than in MH. None of the $S$. aureus isolates from $\mathrm{MH}$ was resistant to cloxacillin. The majority of both CA-UTI and HA-UTI isolates of Staphylococcus epidermidis exhibited high resistance rates to ampicillin (80 and 93\%), cephalothin (60 and $59 \%$ ), cefuroxime (40 and 52\%), clindamycin (60 and $59 \%$ ) and SXT (40 and 60\%).

\section{Discussion}

Our study indicates that $E$. coli is still the most common cause of UTI in the community and hospital settings in Kuwait. Although this finding is similar to a previous study in Kuwait by Helin and Araj in 1986 [11], there is a difference in its prevalence rate, which indicates a decline in the proportion of patients with $E$. coli UTI. Findings similar to ours have been reported in Poland [14], India [15], North America [8, 16], Italy [17], Norway [18] and UK [19]. However, some investigators have shown that the percentage of E. coli as a causative agent of UTI is slowly declining, being replaced by other members of the Enterobacteriaceae and enterococci [20]. In addition, the etiological profile of UTI in the present study shows considerable shift from a previous study, similar to ours, reported from Kuwait in 1986 [11]. In this study, Klebsiella/Enterobacter species and Pseudomonas species were second (18\%) and third (10\%), respectively, in the order of ranking. However, in our study, Candida spp., including $C$. albicans, were the second most common cause of HA-UTI among MKH patients and both HA-UTI and CA-UTI patients of MH. S. agalactiae (GBS) was third in the order of ranking in the same settings in both hos- pitals. These findings may signal a gradual departure from published records of prevalent etiological agents of UTI. The high prevalence of yeast as a cause of UTI may be related to the type of patients, advances in surgical, medical management of the patients as well as excessive use of broad-spectrum antibiotics, high prevalence of diabetes mellitus and obesity in the Kuwaiti population. Other studies have also reported increasing prevalence of UTI caused by Candida spp. A previous report from India [15] indicated that Candida species were the fourth common cause of UTI in their institution. This is supported by the findings in the 2001 ESCMID Study Group Report on a European perspective study on nosocomial UTI, where Candida spp. was the third most commonly isolated micro-organisms from 522 patients [21].

Although CNS and enterococci are common causes of UTI in hospitalized patients in some centers [14, 16, 18], they were not prominent causative agents in our study. Enterococci, in this study, are the fifth commonest etiological agent in hospitalized patients in both hospitals, while CNS was the least common agent. The prevalence rate of enterococci in Kuwait remains unchanged almost two decades after the observation published earlier [11] which showed that enterococci ranked fifth among the pathogens that caused UTI. S. saprophyticus was not a prominent member of the CNS causing UTI in our study; this is in contrast to a report by Nicolle et al. [22] where $95 \%$ of all CNS cultured from urine of symptomatic female outpatients were $S$. saprophyticus.

The high prevalence rate of GBS in CA-UTI and HAUTI at MH and community setting of MKH is an interesting finding worthy of note. Although the ESCMID Study Group Report [21] demonstrated a $1.6 \%$ prevalence rate of GBS in UTI among patients in the non-EU countries, there is scarcely any other report that has published such high prevalence rate of GBS in UTI as observed in our study. The explanation for this observation may be related to the high prevalence $(25 \%)$ of diabetes mellitus among the Kuwaiti population. In a previous study, $36 \%$ of patients with UTI had diabetes mellitus [23] and this disease is a known predisposing factor for GBS infection in adults. Besides, previous experience shows that there is high vaginal colonization rate of pregnant and non-pregnant individuals by GBS in Kuwait [24], and this observation correlates with high prevalence of GBS in UTI [unpubl. obs.].

We are well aware of difficulties in comparing the susceptibility results where different sensitivity methods have been used in various studies. However, we will nonetheless compare our results with those published in other 
parts of the world because the methods employed in this study, Vitek and Kirby-Bauer, correlate well, with the recommended methods. The data presented in this report highlight the problem of bacterial resistance among uropathogens in both hospitalized and community patients with UTI, and is consistent with reports from other centres [15].

In the present study, the high resistance rate of E. coli and other Enterobacteriaceae to ampicillin is alarming. For example, almost three quarters and two thirds of $E$. coli from hospitalized patients in $\mathrm{MKH}$ and $\mathrm{MH}$, respectively, were resistant to ampicillin. This is much higher than the findings reported by other researchers in Italy (36\%) [17], UK (23, 44 and 50\%) [19, 25], USA (43 and $43 \%)[8,16]$, Canada (33\%) [8] and Norway (25\%) [18]. However, a resistance rate of $87 \%$, which is higher than ours, has been reported in India [15].

The Enterobacteriaceae, particularly among the hospitalized patients, in our hospitals exhibited relatively higher resistance rates in general to amoxicillin-clavulanic acid and SXT than those that have been reported elsewhere [16]. In our study and another one [18], nitrofurantoin and amikacin remained very active drugs against most $E$. coli isolates.

As reported in a previous study from Kuwait [11], there is an increase in the resistance rates of E. coli against some antibiotics like ampicillin, gentamicin and nalidixic acid. In general, the level of resistance by E. coli and non- $E$. coli uropathogens against SXT, a drug that is recommended as first-line therapy by the IDSA treatment guidelines [26], is discomforting and cannot be recommended for empirical use in UTI in our country. Resis- tance to fluoroquinolone (ciprofloxacin) in this study is within an acceptable range $(<8 \%)$ for the most common uropathogens in both hospitals. The alternative drugs that have been recommended by the IDSA for empirical treatment of patients with uncomplicated UTI include fluoroquinolones and nitrofurantoin. The results of our study therefore support the use of either of these drugs as a reasonable choice for empiric therapy in uncomplicated UTI, particularly in the community setting.

In contrast to SXT and ampicillin, nitrofurantoin demonstrated the best and most consistent activity against gram-positive isolates in both hospitals. This should reinforce the recommendation for its use in UTI.

\section{Conclusion}

Apart from E.coli, the spectrum of the etiological agents of UTIs in $\mathrm{MKH}$ and $\mathrm{MH}$ and the order of frequency are different when compared with other geographical regions of the world. The bacteria causing UTIs in these hospitals are highly resistant to some of the antibiotics recommended for empiric use in the therapy of UTI in the western countries, but nitrofurantoin and fluoroquinolones remain relatively active against most uropathogens.

\section{Acknowledgement}

We are thankful to Ms Fatima Khodakhast and Mrs Tina Verghese for the collation of record data.

\section{References}

1 Gastmeier P, Kampf G, Weschnewski N, Hauer T, Schulgen G, Schumacher M, Daschner F, Ruden H: Prevalence of nosocomial infections in representative German hospitals. J Hosp Infect 1998;38:37-49.

-2 Foxman B: Recurring urinary tract infection: incidence and risk factors. Am J Public Health 1990;80:331-333.

$>3$ Mobley HL: Virulence of the two primary uropathogens. ASM News 2000;66:403-410.

$\checkmark 4$ Magee JT, Pritchard EL, Fitzgerald KA, Dunstan FDJ, Howard AJ: Antibiotic prescribing and antibiotic resistance in community practice: retrospective study, 1996-1998. BMJ 1999;319:1239-1240.

$\checkmark 5$ Vorland LH, Carlson K, Aalen O: An epidemiological survey of urinary tract infections among outpatients in Northern Norway. Scand J Infect Dis 1985; 17:277-283.
Gupta K, Hooton TM, Wobbe CL, Stamm WE: The prevalence of antimicrobial resistance among uropathogens causing acute uncomplicated cystitis in young women. Int J Antimicrob Agents 1999; 11:305-308.

$>7$ Barrett SP, Savage MA, Rebec MP, Guyot A, Andrews N, Shrimpton SM: Antibiotic sensitivity of bacteria associated with communityacquired urinary tract infections in Britain. $\mathrm{J}$ Antimicrob Chemother 1999;44:359-365.

$>8$ Jones RN, Kugler KC, Pfaller MA, Winokur PL, SENTRY Surveillance Group: Characteristics of pathogens causing urinary tract infections in hospitals in North America: results from the SENTRY Antimicrobial Surveillance Program, 1997. Diagn Microbiol Infect Dis 1999;35:55-63.
9 Vromen M, van der Ven AJ, Knols A, Stobberingh EE: Antimicrobial resistance patterns in urinary tract isolates from nursing home residents. Fifteen years of data reviewed. J Antimicrob Chemother 1999;44:113-116.

10 Zhanel GG, Karlowsky JA, Harding GKM, Carrier A, Mazzulli T, Low DE, Hoban DJ: A Canadian National surveillance study of urinary tract isolates from outpatients: comparison of activities of trimethoprim-sulfamethoxazole, ampicillin, mecillinam, nitrofurantoin and ciprofloxacin. The Canadian Urinary Isolate Study Group. Antimicrob Agents Chemother 2000;44:1089-1092.

$\checkmark 11$ Helin I, Araj GF: Antibiogram of urinary isolates in Kuwait. Scand J Infect Dis 1986;18: 447-450. 
12 Russo TA, Stapleton A, Wenderoth S, Hooton TM, Stamm WE: Chromosomal restriction fragment length polymorphism analysis of Escherichia coli strains causing recurrent urinary tract infections in young women. $\mathrm{J}$ infect Dis 1995; 172:440-445.

13 National Committee on Clinical Laboratory Standards Performance Standards for Antimicrobial Susceptibility Testing: 12th supplement; M100-S12; 2002;22:90-95.

14 Hryniewicz K, Szczypa K, Sulikowska A, Jankowski K, Betlejewska K, Hryniewicz W: Antibiotic susceptibility of bacterial strains isolated from urinary tract infections in Poland. J Antimicrob Chemother 2001;47:773780.

15 Navaneeth BV, Belwadi S, Sughanthi N: Urinary pathogens' resistance to common antibiotics: a retrospective analysis. Trop Doct 2002; 32:20-22.

16 Mathai D, Jones RN, Pfaller MA: SENTRY Participant Group of North America Epidemiology and frequency of resistance among pathogens causing urinary tract infections in 1,510 hospitalized patients: a report from the SENTRY Antimicrobial Surveillance program (North America). Diag Microbiol Infect Dis 2001;40:129-136.
17 Bonadio M, Meini M, Spetaleri P, Gilgi C: Current microbiological and clinical aspects of urinary tract infections. Eur J Urol 2001;40: 439-445.

18 Grude N, Tveten Y, Kristiansen B-E: Urinary tract infections in Norway: bacterial aetiology and susceptibility. A retrospective study of clinical isolates. Clin Microbiol Infect 2001;7: 543-547.

19 Farrell DJ, Morrissey I, De Robeis D, Robbins M, Felmingham D: A UK muticentre study of the antimicrobial susceptibility of bacterial pathogens causing urinary tract infection. J Infect 2003;46:94-100.

20 Gruneberg RN: Changes in urinary pathogens and their antibiotic sensitivities 1971-1992. J Antimicrob Chemother 1994;33(suppl A):18.

21 ESCMID Study Group Report: A European perspective on nosocomial urinary tract infections. I. Report on the microbiology workload, etiology and antimicrobial susceptibility (ESGNI-003 study). Clin Microbiol Infect 2001; 7 523-531.
22 Nicolle LE, Hoban SA, Harding GK: Characterization of coagulase-negative staphylococci from urinary tract specimens. J Clin Microbiol 1983;17:267-271.

23 Dobardzic AM, Dobardzic R: Epidemiological features of complicated UTI in a district hospital of Kuwait. Eur J Epidemiol 1997; 13:465470.

-24 Al-Sweih N, Maiyegun S, Diejomaoh M, Rotimi V, Khodakhast F, Hassan N, George S, Baig S: Streptococcus agalactiae (group B streptococci) carriage in late pregnancy in Kuwait. Med Principles Pract 2004;13:10-14.

25 Winstanley TG, Limb DI, Eggington R, Hancock F: A 10-year survey of the antimicrobial susceptibility of urinary isolates in the UK: the Microbe Base Project. J Antimicrob Chemother 1997;40:591-594.

26 Warren JW, Abrutyn E, Hebel JR, Johnson JR, Schaeffer AJ, Stamm WE: Guidelines for antimicrobial treatment of uncomplicated acute bacterial cystitis and acute pyelonephritis in women. Infectious Diseases Society of America (IDAS). Clin Infect Dis 1999;29:745-758. 